\title{
Stripe-ordered superfluid and supersolid phases in the attractive Hofstadter-Hubbard model
}

\author{
M. Iskin \\ Department of Physics, Koç University, Rumelifeneri Yolu, 34450 Sarlyer, Istanbul, Turkey
}

(Received 27 June 2014; published 12 January 2015)

\begin{abstract}
We use microscopic Bogoliubov-de Gennes formalism to explore the ground-state phase diagram of the single-band attractive Hofstadter-Hubbard model on a square lattice. We show that the interplay between the Hofstadter butterfly and superfluidity breaks spatial symmetry, and gives rise to stripe-ordered superfluid and supersolid phases in large parameter spaces. We also discuss the effects of a trapping potential and comment on the viability of observing stripe-ordered phases with cold Fermi gases.
\end{abstract}

PACS number(s): 03.75.Ss, 03.75.Hh, 67.85.- d, 67.80.kb

Introduction. The so-called Hofstadter butterfly $(\mathrm{HB})$ refers to the fractal energy spectrum of a quantum particle that is confined to move on a two-dimensional tight-binding periodic lattice under the influence of a uniform magnetic flux [1]. There are only two length scales in this simple problem, i.e., the lattice spacing $\ell$ and cyclotron radius $\ell_{B}$, and their competition produce one of the first quantum fractals discovered in physics. This self-similar spectrum exhibits a complex pattern of subbands and minigaps as a function of $\ell / \ell_{B}$, but despite all efforts since its prediction, the limited experimental control over this ratio $\left(\ell / \ell_{B} \ll 1\right.$ even for the largest attainable magnetic fields) has hampered the development of techniques that could probe its effects in natural solid-state crystals. It was only last year that it became possible to observe signatures of this spectrum in graphene-based materials with artificially engineered superlattices under real magnetic fields, however, its full structure largely remains uncharted territory [2-4].

In addition, inspired by the recent realization of artificial gauge fields [5-12], the quest for the Hofstadter spectrum and related phenomena have been revitalized in the cold-atom community [13-20]. For instance, by engineering spatially dependent complex tunneling amplitudes with laser-assisted tunneling and a potential energy gradient, two independent research groups have recently reported compelling evidence for the realization of the Hofstadter-Harper Hamiltonian with neutral rubidium atoms that are loaded into laser-induced periodic potentials [16-18]. Thanks to their greater promise of engineering fully tunable many-body Hamiltonians on demand, even though atomic systems are considered as one of the top candidates for much-desired quantum simulators, currently attainable temperatures in these works are not low enough for resolving the fractal structure of the HB. Nevertheless, captivated by these flourishing efforts, here we study the interplay between the HB, strong interactions, and Zeeman field, and explore ground-state phases of the attractive Hofstadter-Hubbard model [21].

Our main results are highlighted as follows. We find that the cooperation between the HB and superfluid (SF) order breaks the spatial symmetry of the system. This is in accordance with a recent work showing that superfluidity necessarily breaks translation symmetry in a repulsive Hofstadter-BoseHubbard model [22]. In addition, we show that the phase diagrams are dominated by stripe-ordered SF and supersolid (SS) phases which are characterized by their coexisting pairdensity (PDW), charge-density (CDW), and/or spin-densitywave (SDW) orders [21,23-25]. While these phases share some characteristic features of the long-sought Fulde-FerrelLarkin-Ovchinnikov (FFLO or LOFF) phase [26,27], they are not driven by the Zeeman field and have an entirely new physical mechanism. Given that FFLO-like phases are of high demand in condensed-matter, nuclear, and elementary-particle physics [28-32], our findings allude to a new route towards creating them by loading neutral atomic Fermi gases on laser-induced optical lattices under laser-induced gauge fields.

Bogoliubov-de Gennes (BdG) formalism. To achieve these results, we solve the single-band attractive Hofstadter-Hubbard Hamiltonian on a square lattice within the mean-field approximation for the on-site interaction term, i.e., $H=-\sum_{i j \sigma} t_{i j} a_{i \sigma}^{\dagger} a_{j \sigma}-\sum_{i \sigma} \mu_{i \sigma} a_{i \sigma}^{\dagger} a_{i \sigma}+\sum_{i}\left(\Delta_{i} a_{i \uparrow}^{\dagger} a_{i \downarrow}^{\dagger}+\right.$ $\left.\Delta_{i}^{*} a_{i \downarrow} a_{i \uparrow}+\frac{\left|\Delta_{i}\right|^{2}}{g}\right)$, where $a_{i \sigma}^{\dagger}\left(a_{i \sigma}\right)$ creates (annihilates) a $\sigma$ fermion on site $i, t_{i j}$ is the tunneling (hopping) matrix element, and $\mu_{i \uparrow}=\mu-g n_{i \downarrow}-V_{i}+h$ and $\mu_{i \downarrow}=\mu-g n_{i \uparrow}-V_{i}-h$ are effectively the local chemical potentials in the presence of Hartree shifts, confining potential $V_{i}$ and an out-of-plane Zeeman field $h \geqslant 0$. The complex hopping matrix $t_{i j}$ is assumed to connect only the nearest-neighbor sites, i.e., $t_{i j}=t e^{i \theta_{i j}}$ with $t \geqslant 0$ for $i$ and $j$ nearest neighbors and 0 otherwise, and the phase $\theta_{i j}=\left(1 / \phi_{0}\right) \int_{\mathbf{r}_{i}}^{\mathbf{r}_{j}} \mathbf{A}(\mathbf{r}) \cdot d \mathbf{r}$ takes the effects of real external magnetic fields (or artificial gauge fields) into account via the Peierls substitution. Here, A(r) is the corresponding vector potential and $\phi_{0}=2 \pi \hbar / e$ is the magnetic flux quantum. The remaining terms involve the complex SF order parameter $\Delta_{i}=g\left\langle a_{i \uparrow} a_{i \downarrow}\right\rangle$, where $g \geqslant 0$ is the strength of the on-site density-density interaction and $\langle\cdots\rangle$ is a thermal average.

This microscopic Hamiltonian can be diagonalized via the Bogoliubov-Valatin transformation, i.e., $a_{i \sigma}=$ $\sum_{m}\left(u_{m i \sigma} \gamma_{m \sigma}-s_{\sigma} v_{m i \sigma}^{*} \gamma_{m,-\sigma}^{\dagger}\right)$, where $\gamma_{m \sigma}^{\dagger}\left(\gamma_{m \sigma}\right)$ creates (annihilates) a pseudospin $\sigma$ quasiparticle with energy $\epsilon_{m}^{\sigma}$ and wave functions $u_{m i \sigma}$ and $v_{m i \sigma}$, and the resultant $\mathrm{BdG}$ equations can be compactly written as

$$
\sum_{j}\left(\begin{array}{cc}
-t_{i j}-\mu_{i \uparrow} \delta_{i j} & \Delta_{i} \delta_{i j} \\
\Delta_{i}^{*} \delta_{i j} & t_{i j}^{*}+\mu_{i \downarrow} \delta_{i j}
\end{array}\right) \varphi_{m j}^{\sigma}=s_{\sigma} \epsilon_{m}^{\sigma} \varphi_{m i}^{\sigma} .
$$

Here, $\delta_{i j}$ is the Kronecker delta, $\varphi_{m i}^{\uparrow}=\left(u_{m i \uparrow}^{*}, v_{m i \downarrow}^{*}\right)^{\dagger}$ and $\varphi_{m i}^{\downarrow}=\left(v_{m i \uparrow},-u_{m i \downarrow}\right)^{\dagger}$ are the corresponding eigenfunctions for $\epsilon_{m}^{\sigma} \geqslant 0$ eigenvalue, and $s_{\uparrow}=+1$ and $s_{\downarrow}=-1$. Note that the $\mathrm{BdG}$ equations are invariant under the transformations $v_{m i \uparrow} \rightarrow u_{m i \uparrow}^{*}, u_{m i \downarrow} \rightarrow-v_{m i \downarrow}^{*}$, and $\epsilon_{m \downarrow} \rightarrow-\epsilon_{m \uparrow}$, and 
therefore, it is sufficient to solve only for $u_{m i} \equiv u_{m i \uparrow}, v_{m i} \equiv$ $v_{m i \downarrow}$, and $\epsilon_{m} \equiv \epsilon_{m}^{\uparrow}$ as long as all solutions with positive and negative $\epsilon_{m}$ are kept. For instance, $\Delta_{i}$ is given by $\Delta_{i}=-g \sum_{m} u_{m i} v_{m i}^{*} f\left(\epsilon_{m}\right)$, where $f(x)=1 /\left[e^{x /\left(k_{B} T\right)}+1\right]$ is the Fermi function with $k_{B}$ the Boltzmann constant and $T$ the temperature, and it has to be determined self-consistently with $\mu$ and $h$ such that the total number of $\sigma$ fermions satisfies $N_{\sigma}=\sum_{i} n_{i \sigma}$. Here, $0 \leqslant n_{i \sigma}=\left\langle a_{i \sigma}^{\dagger} a_{i \sigma}\right\rangle \leqslant 1$ is the number of $\sigma$ fermions on site $i$ given by $n_{i \uparrow}=\sum_{m}\left|u_{m i}\right|^{2} f\left(\epsilon_{m}\right)$ and $n_{i \downarrow}=\sum_{m}\left|v_{m i}\right|^{2} f\left(-\epsilon_{m}\right)$. When $\theta_{i j}=0$, it is generally accepted that the mean-field description provides a qualitative understanding of the ground state $[33,34]$, and here we include both $\theta_{i j}$ and $V_{i}$ exactly into the mean-field formalism without relying on further approximations.

Without losing generality, we choose Landau gauge for the vector potential, i.e., $\mathbf{A}(\mathbf{r}) \equiv(0, B x, 0)$, leading to a uniform magnetic flux $\Phi=B \ell^{2}$ (per unit cell), where $\ell$ is the lattice spacing. Denoting $(x, y)$ coordinates of site $i$ by $(n \ell, m \ell)$, this gauge simply implies $\theta_{i j}=0$ and $\theta_{i j}= \pm 2 \pi n \phi$ for links along the $x$ and $y$ directions, respectively, where $\phi=\Phi /\left(2 \pi \phi_{0}\right)$ characterizes the competition between the lattice spacing $\ell$ and magnetic length scale $\ell_{B}=\sqrt{\hbar /(e B)}$. In this Rapid Communication, we only consider $\phi=p / q$ ratios, where $p$ and $q$ are coprimes, for which the exact noninteracting single-particle excitation spectrum $\varepsilon(\phi)$ vs $\phi$ is known as the HB [1]. While $\phi$ remains $\ll 1$ for typical electronic crystals, even for the largest $B$ field that is attainable in a laboratory, it can in principle be tuned at will for atomic systems via artificial gauge fields. The fractal structure of the spectrum is expected to have drastic effects on the many-body problem which is our main motivation.

Ground-state phases. In order to explore the possible phases, let us set $V_{i}=0$ and consider a uniform $45 \ell \times 45 \ell$ square lattice, which is large enough to construct the thermodynamic phase diagrams for $\phi=\{0,1 / 6,1 / 4\}$. The Hartree shifts are neglected for simplicity [35]. It turns out that the BdG equations allow for multiple solutions, especially for the polarized many-body phases, and therefore, it is essential to verify the (meta)stability of the solutions [36].

Depending on the spatial profiles of $\left|\Delta_{i}\right|, n_{i \uparrow}$, and $n_{i \downarrow}$, we distinguish the single-particle phases from the many-body ones using the following scheme. When $h / g$ is sufficiently high that $\Delta_{i} \rightarrow 0\left(\left|\Delta_{i}\right|<10^{-3} t\right.$ in numerics $)$, the ground state can be a $\sigma$-vac phase which is a vacuum of $\sigma$ component, a $\sigma-I(m / n)$ phase which is a band insulator of $\sigma$ component with uniform $n_{i \sigma}=m / n$, a $\sigma-N$ phase which is a normal $\sigma$ component, or an $\uparrow \downarrow-P N$ phase which is a polarized normal mixture of $\uparrow$ and $\downarrow$ components. On the other hand, when $h / g$ is sufficiently low, the ground state can be characterized according to Table I,

TABLE I. The uniform-SF (U-SF), striped-SF (S-SF), and striped-SS (S-SS) phases can be distinguished by their coexisting order parameters.

\begin{tabular}{lccc}
\hline \hline Phase & $\left|\Delta_{i}\right|$ & $n_{i \uparrow}+n_{i \downarrow}$ & $n_{i \uparrow}-n_{i \downarrow}$ \\
\hline U-SF & Uniform & Uniform & 0 \\
S-SF & PDW & 1 & 0 \\
S-SS & PDW & CDW & 0 \\
\hline \hline
\end{tabular}
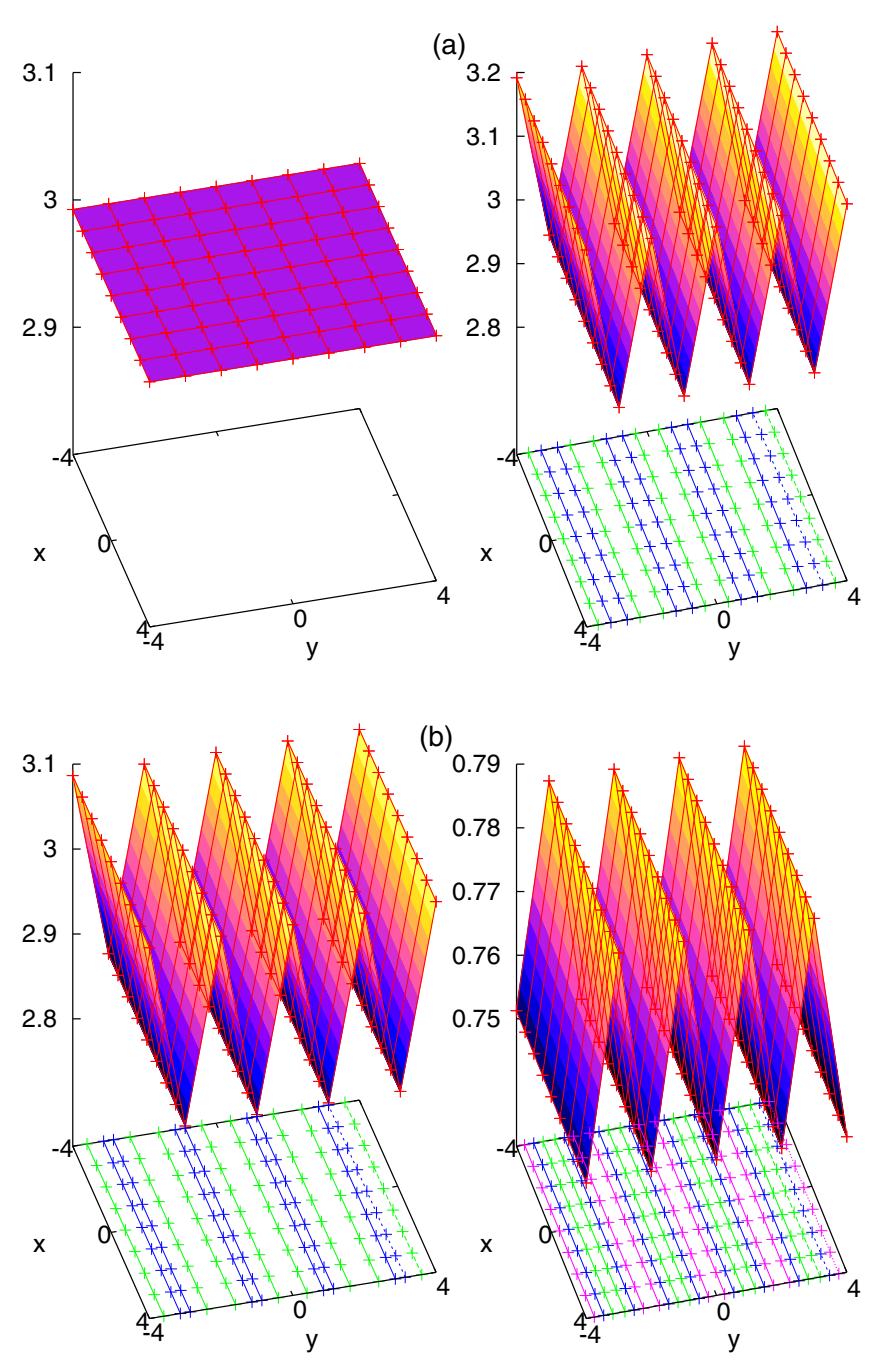

FIG. 1. (Color online) (a) Typical $\left|\Delta_{i}\right| / t$ profiles are shown for the U-SF (left) and S-SF phases (right), where $\phi_{\uparrow}=\phi_{\downarrow}=0$ and $\phi_{\uparrow}=$ $\phi_{\downarrow}=1 / 4$, respectively, and $\mu=0$ corresponding to a uniformly halffilled lattice. (b) Typical $\left|\Delta_{i}\right| / t$ (left) and $n_{i \uparrow}+n_{i \downarrow}$ (right) profiles are shown for the S-SS phase, where $\phi_{\uparrow}=\phi_{\downarrow}=1 / 4$ and $\mu=-t$. Here, $(x, y)$ are in units of $\ell$, and we set $h=0$ and $g=7 t$ in all figures.

and typical $\left|\Delta_{i}\right|$ and $n_{i \uparrow}+n_{i \downarrow}$ profiles are illustrated in Fig. 1 for the uniform-SF, striped-SF, and striped-SS phases.

Thermodynamic phase diagrams. In Fig. 2, we present the $\phi=1 / 4$ phase diagrams for $\mu=0$ in Fig. 2(a) and $\mu=-t$ in Fig. 2(b). The $\mu=0$ case is very special since it corresponds to a half-filled lattice with particle-hole symmetry, where $n_{i \uparrow}+n_{i \downarrow}=1$ independently of $i$, no matter what the rest of the parameters are. In comparison to the $\phi=0$ diagrams which consist only of $\uparrow \downarrow-P N$, uniform-SF, and polarized-SF regions, we find that the $\phi=1 / 4$ diagrams have much richer structure involving large regions of stripe-ordered phases. To understand the physical origin of the resultant phase diagrams and stripe order, next we discuss the analytically tractable highand low- $h / g$ limits.

When $h / g$ is sufficiently high, we can directly read off the ground state of the $\sigma$ component from the HB for any given $\phi$. For $\phi=1 / 4$, the energy spectrum consists of four bands: the $\sigma$ component is a $\sigma$-vac for $\mu_{\sigma} \lesssim-2.83 t$, a $\sigma-N$ for 

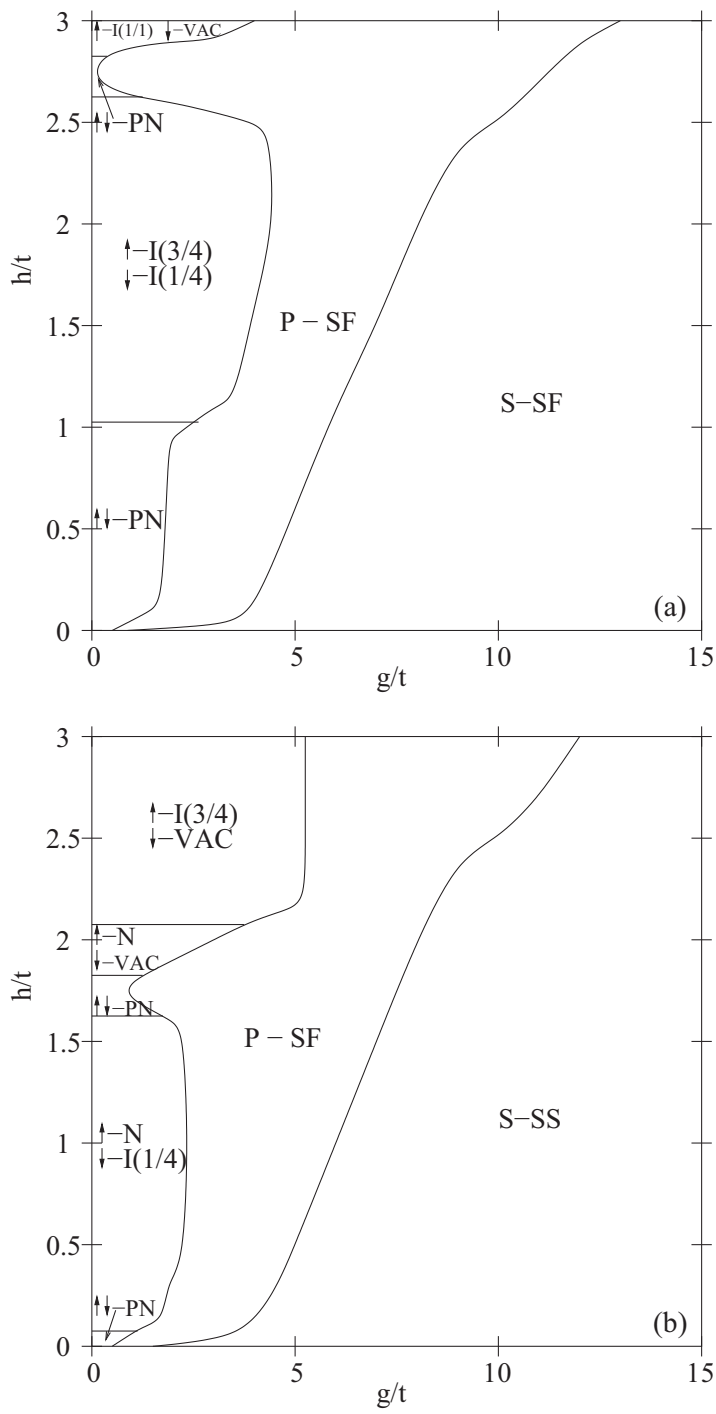

FIG. 2. The ground-state phase diagrams are shown for $\mu=0$ in (a) and $\mu=-t$ in (b), when $\phi=p / q=1 / 4$, and the solid lines are guides to the eye.

$-2.83 t \lesssim \mu_{\sigma} \lesssim-2.61 t$, a $\sigma-I(1 / 4)$ for $-2.61 t \lesssim \mu_{\sigma} \lesssim$ $-1.082 t$, a $\sigma-N$ for $-1.082 t \lesssim \mu_{\sigma} \lesssim 1.082 t$, a $\sigma-I(3 / 4)$ for $1.082 t \lesssim \mu_{\sigma} \lesssim 2.61 t$, a $\sigma-N$ for $2.61 t \lesssim \mu_{\sigma} \lesssim 2.83 t$, and a $\sigma-I(1 / 1)$ for $2.83 t \lesssim \mu_{\sigma}$. Using $\mu_{\uparrow}=\mu+h$ and $\mu_{\downarrow}=\mu-h$, the high- $h / g$ structure of Fig. 2 immediately follows. As $h / g$ gets smaller, the $I$ and $N$ phases must pave the way to ordered many-body ones, as increasing the strength of the pairing energy eventually makes them energetically less favourable. For $\phi=0$, we confirmed that the $\uparrow \downarrow-P N$ to polarized-SF phase transition boundary $g\left(h_{c}\right)$ is a monotonic function of $h$, which is simply because the noninteracting system has a very simple band structure with cosine dispersions. However, since the density of single-particle states is dramatically affected by the fractal band structure, the transition boundary $g\left(h_{c}\right)$ becomes a complicated function of $h$ for finite $\phi$. For instance, we find a sizable hump in Fig. 2(a) around $h \approx 2.7 t$ and another one in Fig. 2(b) around $h \approx 1.7 t$, the peak locations of which coincide intuitively with the $\uparrow \downarrow-P N$ regions that are sandwiched between vac and/or $I$.
On the other hand, when $h / g$ is sufficiently small, the ground state is expected to be an ordered many-body phase with no polarization. In sharp contrast to the $\phi=0$ case where uniform-SF is stable for any $\mu$, we show in Fig. 2 that striped-SF and striped-SS are, respectively, stable for $\mu=0$ and $\mu=-t$ when $\phi=1 / 4$. Note that since $\mu=0$ corresponds to half filling for any $\phi$, the unpolarized ground states necessarily have uniform fillings, i.e., $n_{i \uparrow}=n_{i \downarrow}=1 / 2$. Therefore, in the low $h / g$ limit, while only $\left|\Delta_{i}\right|$ is allowed to have spatial modulations in Fig. 2(a), both $\left|\Delta_{i}\right|$ and $n_{i \sigma}$ modulates in Fig. 2(b).

The stripe order is a result of the HB: for a given $\phi$, the spectrum consists of $q$ bands in the first magnetic Brillouin zone within which each $\mathbf{k}$ state is $q$-fold degenerate. Therefore, not only intra- and interband pairings but also pairings with both zero and a set of nonzero center-of-mass momenta are allowed [21,24], leading to a nonuniform $\left|\Delta_{i}\right|$ with spatially periodic modulations, e.g., a PDW order [25]. The directions of center-of-mass momenta determine the direction of modulations, making it gauge dependent, e.g., $y$ direction in Fig. 1. When the striped-PDW order is sufficiently large, it drives an additional striped-CDW order in the total fermion filling, giving rise to striped-SS phases. We emphasize that the instability towards striped-PDW phases discussed in this Rapid Communication is driven by $\phi \neq 0$ even when $h=0$, and they cannot formally be identified with the FFLO-like nonstriped PDW phases which are driven by $h \neq 0$ and are characterized by cosinelike sign-changing $\left|\Delta_{i}\right|$ oscillations along a spontaneously chosen direction.

It is clearly the cooperation between $\phi$ and $g$ that is responsible for the broken spatial symmetry and appearance of stripe order, causing much more prominent stripes for intermediate $g$ at a given $h$. Depending on whether $q$ is odd or even, $\left|\Delta_{i}\right|$ modulation has a spatial period of $q$ or $q / 2$ lattice sites, respectively. The stripe order gradually fades away with increasing $g$, however, it survives even in the $g \gg W$ limit with $W$ the single-particle energy bandwidth, as long as $g / t$ is finite. Note in this limit that the physics is eventually determined by the two-body bound states, i.e., Cooper pairs become bosonic dimers, and unless $g / t \rightarrow \infty$, the dimer-dimer interaction ( $\left.g_{d d} \sim t^{2} / g\right)$ is finite. Such weakly repulsive dimers can effectively be described by the Hofstadter-Bose-Hubbard model, where superfluidity has recently been shown to break translation symmetry in the weakly interacting limit [22].

In fact, all of our numerical results fit quite well with

$$
\left|\Delta_{i}\right|=\left|\Delta_{0}\right|+\left|\Delta_{1}\right| \cos \left(4 \pi \phi i_{y} / \ell+\varphi\right),
$$

in the entire unpolarized region (U-SF, S-SF, and S-SS). Here, $\left|\Delta_{0}\right| \approx\left(g / 2-4 t^{2} / g\right) \sqrt{n(2-n)}$ for any $\mu$ determined by the total average filling $n,\left|\Delta_{1}\right| \approx t^{2} / g$ for $\mu \approx 0$ (which becomes exact only for $\mu=0$ in the $g / t \rightarrow \infty$ limit), $i_{y}$ is the $y$ coordinate of site $i$, and $\varphi$ is a constant phase shift set by the origin. The microscopic origin of this expression can be best understood in the ideal-dimer limit, where $t_{d} \approx 2 t^{2} / g$ and $\phi_{d}=p_{d} / q_{d}$, respectively, are their effective hopping and gauge field. Here, $p_{d}=2 p(p)$ and $q_{d}=q(q / 2)$ for odd (even) $q$. Since the HB for dimers is $q_{d}$-fold degenerate, their ground state has contributions from all degenerate $k_{y d}=$ $\left\{0, \pm 2 \pi \phi_{d} f / \ell\right\}$ momenta where $f=1, \ldots, q_{d}-1$, such that $\Psi_{i d}=c_{0}+\sum_{f} c_{f} \cos \left(2 \pi \phi_{d} f i_{y} / \ell\right)$, where $c_{f}$ are complex 
variational parameters. However, unlike atomic bosons where all of the degenerate states have equal weight, dimer bosons are fermion pairs and the number of ways of creating them with $k_{y d}=k_{y \uparrow}+k_{y \downarrow}$ momentum depends on $f$ and $\phi$, e.g., there are $2(q-f-1)+1$ ways of intraband pairing when $q$ is even. Thus, this analysis shows that higher $\left|k_{y d}\right|$ states contribute less and less, forming a perturbative series. In addition, our fit, Eq. (2), suggests that the first-order $(f=1)$ correction is already much smaller than the zeroth-order ( $f=0$ ) one, and that the $f \geqslant 2$ terms are negligible.

When $\phi$ is increased from 0 , we find that the transition from an unpolarized to a polarized ordered phase occurs at a lower $h$ for any given $g$. This is a consequence of smaller $W$ : As $\phi$ increases from 0 to $1 / 6$ to $1 / 4$, then $W$ shrinks from $8 t$ to $6.15 t$ to $5.65 t$, making it possible to polarize the ground state with a smaller and smaller $h$. In Fig. 2, the polarized region is dominated mainly by what we call the polarizedSF phase which is characterized by striped and/or nonstriped PDW and/or SDW orders.

Trapped systems. Next, we assume a harmonic confinement and choose $V_{i}=\alpha\left|\mathbf{r}_{\mathbf{i}}\right|^{2}$ with strength $\alpha=0.01 t / \ell^{2}$. For illustration purposes, in Fig. 3 we show typical trap profiles for a cut along the $y$ direction when $x=0, \phi=1 / 4, \mu=0$, and $h=t$. Note that the system is completely unpolarized for $g=7 t$ with no SDW order, which is consistent with the phase diagrams shown in Fig. 2. When $g / t$ is sufficiently small, the minigaps of the HB give rise to a wedding-cake structure with spatially flat $n_{i \sigma}$ regions at integer multiples of $1 / 4$ fillings, but only the $n_{i \uparrow} \approx 0.25$ and $n_{i \downarrow} \approx 0$ region clearly survives in these figures. In addition, while the CDW order is mostly washed out and barely visible for $g=6 t$ and $g=7 t$, the PDW and SDW orders are large and conspicuous in these figures (and can be much larger depending on the parameters, especially for large $q$ ), suggesting that PDW and/or SDW features may furnish clearest and direct evidence in trapped systems. It is also pleasing to see that the valleys of the PDW and CDW orders and peaks of the SDW order coincide when they coexist.

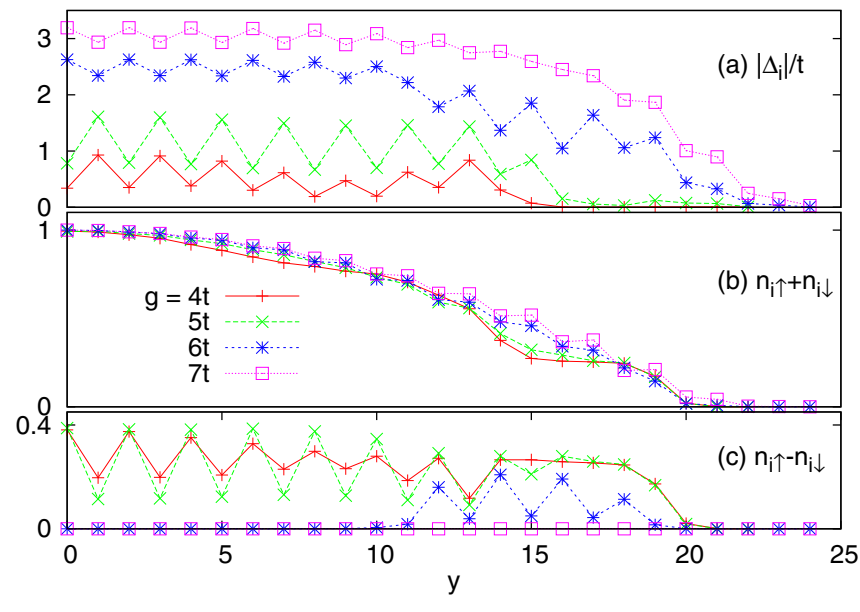

FIG. 3. (Color online) The trap profiles are shown for a cut along the $y$ direction (in units of $\ell$ ) when $x=0, p / q=1 / 4, \mu=0$, and $h=t$.

Conclusions. To summarize, motivated by the thrive of cold-atom experiments with artificial gauge fields, we analyzed ground-state phases of the attractive Hofstadter-Hubbard model for both thermodynamic and trapped systems. Our main finding is that the interplay between the $\mathrm{HB}$ and SF order breaks spatial symmetry, and that the phase diagrams are dominated by stripe-ordered SF and SS phases which can be distinguished by their coexisting PDW, CDW, and/or SDW orders. Such PDW superconductivity is relevant in a diverse range of systems [25,28,30], and given our promising results for atomic Fermi gases, we encourage further research in this direction with different lattice geometries, gauge fields, etc., in particular, the beyond mean-field ones.

Acknowledgments. This work is supported by the Marie Curie IRG Grant No. FP7-PEOPLE-IRG-2010-268239, TÜBİTAK 1001-114F232. The author thanks A. L. Subaş1 and R. O. Umucalilar for discussions.
[1] D. R. Hofstadter, Phys. Rev. B 14, 2239 (1976).

[2] C. R. Dean et al., Nature (London) 497, 598 (2013).

[3] L. A. Ponomarenko et al., Nature (London) 497, 594 (2013).

[4] See also, U. Kuhl and H.-J. Stöckmann, Phys. Rev. Lett. 80, 3232 (1998), for a microwave realization.

[5] V. Galitski and I. B. Spielman, Nature (London) 494, 49 (2013).

[6] J. Dalibard, F. Gerbier, G. Juzeliŭnas, and P. Öhberg, Rev. Mod. Phys. 83, 1523 (2011).

[7] J.-Y. Zhang, S.-C. Ji, Z. Chen, L. Zhang, Z.-D. Du, B. Yan, G.-S. Pan, B. Zhao, Y.-J. Deng, H. Zhai, S. Chen, and J.-W. Pan, Phys. Rev. Lett. 109, 115301 (2012).

[8] P. Wang, Z.-Q. Yu, Z. Fu, J. Miao, L. Huang, S. Chai, H. Zhai, and J. Zhang, Phys. Rev. Lett. 109, 095301 (2012).

[9] L. W. Cheuk, A. T. Sommer, Z. Hadzibabic, T. Yefsah, W. S. Bakr, and M. W. Zwierlein, Phys. Rev. Lett. 109, 095302 (2012).

[10] C. Qu, C. Hamner, M. Gong, C. Zhang, and P. Engels, Phys. Rev. A 88, 021604(R) (2013).
[11] Z. Fu, L. Huang, Z. Meng, P. Wang, X.-J. Liu, H. Pu, H. Hu, and J. Zhang, Phys. Rev. A 87, 053619 (2013).

[12] R. A. Williams, M. C. Beeler, L. J. LeBlanc, K. Jiménez-García, and I. B. Spielman, Phys. Rev. Lett. 111, 095301 (2013).

[13] K. Jiménez-García, L. J. LeBlanc, R. A. Williams, M. C. Beeler, A. R. Perry, and I. B. Spielman, Phys. Rev. Lett. 108, 225303 (2012).

[14] J. Struck, C. Ölschläger, M. Weinberg, P. Hauke, J. Simonet, A. Eckardt, M. Lewenstein, K. Sengstock, and P. Windpassinger, Phys. Rev. Lett. 108, 225304 (2012).

[15] C. J. Kennedy, G. A. Siviloglou, H. Miyake, W. C. Burton, and W. Ketterle, Phys. Rev. Lett. 111, 225301 (2013).

[16] M. Aidelsburger, M. Atala, M. Lohse, J. T. Barreiro, B. Paredes, and I. Bloch, Phys. Rev. Lett. 111, 185301 (2013).

[17] H. Miyake, G. A. Siviloglou, C. J. Kennedy, W. C. Burton, and W. Ketterle, Phys. Rev. Lett. 111, 185302 (2013).

[18] C. Chin and E. J. Mueller, Physics 6, 118 (2013). 
[19] D. Cocks, P. P. Orth, S. Rachel, M. Buchhold, K. Le Hur, and W. Hofstetter, Phys. Rev. Lett. 109, 205303 (2012).

[20] L. Wang, H.-H. Hung, and M. Troyer, Phys. Rev. B 90, 205111 (2014).

[21] Hui Zhai, R. O. Umucalılar, and M. O. Öktel, Phys. Rev. Lett. 104, 145301 (2010).

[22] S. Powell, R. Barnett, R. Sensarma, and S. Das Sarma, Phys. Rev. Lett. 104, 255303 (2010).

[23] Although the vortex lattice instability in the two-dimensional Hofstadter-Hubbard lattice model has been known for a long time (see, for instance, [21]), a cosine-shaped PDW instability has recently been found in a somewhat related model (a continuum three-dimensional system in the lowest-Landau-level limit with an anisotropic trap) [24]. While our striped-PDW order is a direct result of the HB spectrum, the PDW instability that is found in Ref. [24] is driven by the specific form of the effective one-dimensional (1D) interaction obtained after mapping the system to an effectively $1 \mathrm{D}$ one in $\mathbf{k}$ space.

[24] R. Wei and E. J. Mueller, Phys. Rev. Lett. 108, 245301 (2012).

[25] D. F. Agterberg and H. Tsunetsugu, Nat. Phys. 4, 639 (2008).

[26] P. Fulde and R. Ferrell, Phys. Rev. 135, A550 (1964).

[27] A. I. Larkin and Y. N. Ovchinnikov, Sov. Phys. JETP 20, 762 (1965).
[28] R. Casalbuoni and G. Nardulli, Rev. Mod. Phys. 76, 263 (2004).

[29] M. Boninsegni and N. V. Prokofev, Rev. Mod. Phys. 84, 759 (2012).

[30] R. Anglani, R. Casalbuoni, M. Ciminale, N. Ippolito, R. Gatto, M. Mannarelli, and M. Ruggieri, Rev. Mod. Phys. 86, 509 (2014).

[31] S. A. Kivelson, I. P. Bindloss, E. Fradkin, V. Oganesyan, J. M. Tranquada, A. Kapitulnik, and C. Howald, Rev. Mod. Phys. 75, 1201 (2003).

[32] Tao Wu et al., Nature (London) 477, 191 (2011).

[33] T. Esslinger, Annu. Rev. Condens. Matter Phys. 1, 129 (2010).

[34] A. Koetsier, D. B. M. Dickerscheid, and H. T. C. Stoof, Phys. Rev. A 74, 033621 (2006).

[35] We neglected them because not only do the self-consistent solutions converge much faster, the solutions are also much easier to interpret. In addition, since none of the PDW, CDW, SDW, and VL instabilities are driven by these shifts, our meanfield results already pave the way to quantitative understanding of the possible ground states of the model.

[36] However, there is no multiple solution problem in the low- $h / g$ limit, where we find unique U-SF, S-SF, or S-SS solutions, depending on whether $\mu=0$ or not. 